\title{
Persistence of poor sleep predicts the severity of the clinical condition after 6 months of standard treatment in patients with eating disorders
}

\author{
Caterina Lombardo ${ }^{\text {a, } *}$, Gemma Battagliese ${ }^{\text {a }}$, Carmela Venezia ${ }^{\text {a }}$, Vito Salvemini ${ }^{\text {b }}$ \\ a Department of Psychology, Sapienza University of Rome, Via dei Marsi 78, 00185 Rome, Italy \\ ${ }^{b}$ Multidisciplinary Section for Eating Disorders, Traumatological and Orthopaedic Hospital "Andrea Alesini", Via San Nemesio 21, 00144 Rome, Italy
}

\section{A R T I C L E I N F O}

\section{Article history:}

Received 12 September 2014

Received in revised form 10 February 2015

Accepted 19 March 2015

Available online 27 March 2015

\section{Keywords:}

Eating disorders

Poor sleep

Depression

Mediation

Comorbidity

\begin{abstract}
A B S T R A C T
Clinical evidence suggests that eating disorder (ED) patients experience poor sleep even if they rarely complain of it. However, direct empirical evidence supporting this relationship is still sparse. In order to provide direct evidence, poor sleep, severity of the ED symptoms and depression were obtained in 562 ED patients at treatment admission (T0). For 271 patients out of them, data were also available after 6 months of standard treatment (T1). Results evidence that at T0 poor sleep predicts severity of ED symptoms through the mediation of depression. Persistence of poor sleep at T1 directly predicts the severity of the ED symptoms both directly and through the mediation of depression. These findings suggest that the treatment of ED may benefit from addressing poor sleep since its presence and persistence increase comorbidity and attrition to the standard treatment.
\end{abstract}

(c) 2015 Elsevier Ltd. All rights reserved.

\section{Introduction}

Patients with eating disorder (ED) frequently experience poor sleep (e.g. Crisp, Stonehill, \& Fenton, 1971) but direct epidemiological evidence of the co-occurrence of anorexia nervosa (AN) or bulimia nervosa (BN) and insomnia is almost lacking. Indirect evidence indicates that poor sleep predicts obesity in different populations (e.g. Patel, 2009; Patel et al., 2008; Van Cauter \& Knutson, 2008; Van der Berg et al., 2008) and that the experimental induction of hunger in laboratory produces increased nocturnal wakefulness and reduced slow wave sleep (Ohno \& Sakurai, 2008). The sleep disruptive effect of hunger is also consistent with results of polysomnographic studies that provide evidence of reduced sleep efficiency, increased wake time, and/or increased stage 1 NREM sleep in restrictive type AN compared with control groups (Lauer \& Krieg, 2004).

On the behavioral level, direct evidence of this link is still sparse. Recently, Bayard and Dauvilliers (2013) reported that EDs, especially $\mathrm{BN}$, are more prevalent in patients with narcolepsy with cataplexy as compared to controls. Few community studies (Makino, Hashizume, Yasushi, Tsuboy, \& Dennerstein, 2006; Soares et al., 2011), using a survey approach, provide evidence of a positive association between self-reported poor sleep and severity of ED symptoms. A recent study (Lombardo et al., 2013), conducted in a community sample,

\footnotetext{
* Corresponding author at: Via dei Marsi 78, 00185 Rome, Italy. Tel.: + 390649917529 E-mail addresses: caterina.lombardo@uniroma1.it (C. Lombardo), gemma.battagliese@uniroma1.it (G. Battagliese), carmen.venez@alice.it (C. Venezia), salvemini.vito@aslrmc.it (V. Salvemini).
}

demonstrated that insomnia has a strong indirect link, mediated by depression, and a weak direct link with the severity of eating disorder symptoms.

To the best of our knowledge, only a study by Kim and colleagues (Kim et al., 2010) assessing the relationship between sleep problems and eating disorder symptoms in a clinical sample has been published to date. Results evidence that sleep disturbances were highly prevalent (50.3\%) among $400 \mathrm{ED}$ patients. In addition, the general symptomatological condition of those patients who also had sleep disturbances as compared to those who did not complain of them was more severe. The study, however, does not allow exclusion that the association found is mediated by depression.

The present study aims to:

1) replicate Kim and colleagues' findings evaluating also the mediation of depression;

2) evaluate whether poor sleep predicts the severity of the clinical condition after 6 months of standard treatment.

\section{Materials and methods}

\subsection{Participants and procedure}

Data examining the clinical files of 623 outpatients asking for treatment at an outstanding multidisciplinary public clinic in Rome, from March 1995 to March 2013 were collected. According to the steppedcare protocol used, at admission, each patient receives psychiatric, nutritional and psychological evaluations by experts of the service. 
Evaluations are repeated in different time points during therapy. Due to the aim of the present study, data regarding 562 female patients (mean age $=33.53 ;$ SD 12.0 , range $15-75$ years) were obtained from the data file where they are routinely recorded. The information extracted includes: the DSM-IV-TR diagnosis; the nutritional status $\left(\mathrm{BMI}=\right.$ weight/height $\left.{ }^{2}\right)$; the scores obtained in the questionnaires described below. The same information was extracted for the second evaluation, after around 6 months of standard therapy. In this case, data were available only for 271 patients.

As the study is an archive research, following the guidelines and standards of the Helsinki declaration (2013, section 32), although in absence of signed consent forms, the study was submitted for approval to the Ethics Committee of the Department of Psychology.

\subsection{Measures}

Severity of eating disorder (ED) symptoms was assessed through the Eating Disorder Inventory (EDI-2, Garner, 1991), a 91 item self-report questionnaire that consists of 11 subscales assessing both the ED symptoms and several behavioral and psychological characteristics known to be associated with these disorders. Responses are given on a 6 point frequency scale ranging from 1 to 6 and scored assigning 0 to steps 1 (never), 2 (rarely) and 3 (sometimes); step 1 to step 4 (often); step 2 to step 5 (usually), and step 3 to step 6 (always). For the present study, only the following subscales were selected: Body Dissatisfaction (BD); Drive for Thinness (DT); and Bulimia (BU).

Depression and sleep problems were measured through the SCL-90 (e.g. Derogatis, Lipman, \& Covi, 1973), a 90 item self-report questionnaire assessing the severity of psychopathological symptoms that occurred during the week preceding the evaluation. Answers are given on a scale ranging from 1 (not at all) to 5 (extremely). It allows computing the scores on several psychopathological scales (e.g. depression and sleep problems) and it also gives a composite score of general symptomatological severity (GSI). Validity and reliability have been evidenced both in clinical (Horowitz, Rosenberg, Baer, Ureno, \& Villasenor, 1988) and nonclinical samples (Derogatis, Rickels, \& Rock, 1976). Due to the aim of the present study the depression and sleep problem scales were used.

\section{Results}

Analyses were conducted using SPSS 19 software for Windows.

The frequency distribution of patients across diagnoses and the descriptive statistics of the sample are reported in Table 1. The table also reports results of the one-way ANOVAs comparing diagnostic groups on age, BMI, BD, DT, and BU scores.

Groups were also compared with respect to depression and sleep problem scores. Results evidenced no significant difference.

The relationship between eating disorder symptoms and poor sleep considering the mediation of depression was evaluated using the SPSS macro called PROCESS, proposed by Preacher and Hayes (2008). The models examined are the following:

1) 3 models that considered poor sleep measured at T0 as predictor, the Drive for Thinness, Body Dissatisfaction and Bulimia scales all measured at T0 respectively as criterion, and depression at T0 as mediator and controlling for age.

2) 3 models that considered poor sleep measured at T0 as predictor, the Drive for Thinness, Body Dissatisfaction and Bulimia scales all measured at T1 respectively as criterion, and depression at T0 as mediator and controlling for age.

3) 3 models that considered as predictor an index of persistence of poor sleep obtained by summing up scores obtained in the sleep problem scale of the SCL-90 at T0 and those obtained at T1. The Drive for Thinness, Body Dissatisfaction and Bulimia scores at T1 were again respectively the criterion variables and depression measured at T0 as mediator. Age was always controlled for.

All measures were standardized before running PROCESS. A bootstrapping procedure was used to obtain estimates and confidence intervals around the indirect effects.

The total model is statistically significant for DT ( $\mathrm{R}$-squared $=0.104$; $\left.\mathrm{F}_{(3,531)}=20.51 ; \mathrm{p}<.001\right)$, for $\mathrm{BD}\left(\mathrm{R}\right.$-squared $=0.101 ; \mathrm{F}_{(3,531)}=19.93$; $\mathrm{p}<.001)$ and for $\mathrm{BU}\left(\mathrm{R}\right.$-squared $\left.=0.134 ; \mathrm{F}_{(3,531)}=27.44 ; \mathrm{p}<.001\right)$. In all cases, only the indirect paths are significant ( $\mathrm{b}$ ranging from .265 to .521 ) while the direct paths are not. The mediational models that tested the effects of poor sleep measured at T0 on the three measures of eating disorder symptoms (namely DT, BD, BU) measured at T1 considering the mediation of depression (measured at T0), using age as covariate yielded a statistically significant total effects for all the dependent variables considered (DT: R-squared $=0.056 ; \mathrm{F}_{(3,247)}=4.84 ; \mathrm{p}=.003 ; \mathrm{BD}$ : R-squared $=0.077 ; \mathrm{F}_{(3,247)}=6.90 ; \mathrm{p}<.001 ; \mathrm{BU}$ : R-squared $=0.048$; $\left.\mathrm{F}_{(3,247)}=4.17 ; \mathrm{p}=.006\right)$. As in the previous analyses, only the indirect paths are significant (b ranging from .145 to .471 ).

An index of persistence of poor sleep was computed by summing up the scores on the sleep scale at T0 and those obtained at T1. Three mediational models were tested, considering the effect of persistence of poor sleep as predictor, depression measured at T0 as mediator and the three measures of eating disorder symptoms measured at T1 as criterion. Age was always used as covariate. The total model is statistically significant for DT (R-squared $=0.097 ; \mathrm{F}_{(3,231)}=8.31 ; \mathrm{p}<.001$ ), for $\mathrm{BD}\left(\mathrm{R}\right.$-squared $\left.=0.088 ; \mathrm{F}_{(3,231)}=7.39 ; \mathrm{p}<.001\right)$ and for $\mathrm{BU}$ (R-squared $\left.=0.088 ; \mathrm{F}_{(3,231)}=7.46 ; \mathrm{p}<.001\right)$. The direct and indirect significant paths (with the corresponding b) for each model are reported in Fig. 1. Depression resulted a significant mediator except for BU. The covariate resulted non-significant in all analyses.

The median of the sleep problem scale of the SCL-90-R at T0 was 1.67; and the median at T1 was 1 . The following sleep subgroups were obtained dividing participants according to both medians:

1) Good Sleep Group (GSG): patients scoring below the median of the sample at both T0 and T1 assessments: $\mathrm{N}=67$;

Table 1

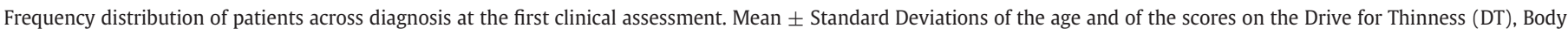

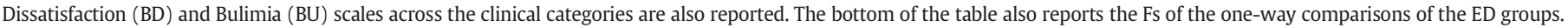
**indicate p values lower than .001. Different letters next to each SD indicate significant differences at Scheffé post-hoc test.

\begin{tabular}{|c|c|c|c|c|c|c|}
\hline & Frequency & $\begin{array}{l}\text { Mean } \\
\text { Age } \pm \text { SD }\end{array}$ & $\begin{array}{l}\text { Mean } \\
\text { BMI } \pm \text { SD }\end{array}$ & $\begin{array}{l}\text { Mean } \\
\mathrm{DT} \pm \mathrm{SD}\end{array}$ & $\begin{array}{l}\text { Mean } \\
\mathrm{BD} \pm \mathrm{SD}\end{array}$ & $\begin{array}{l}\text { Mean } \\
\mathrm{BU} \pm \mathrm{SD}\end{array}$ \\
\hline AN & 67 & $25.59 \pm 8.4^{\mathrm{b}}$ & $16.24 \pm 1.3^{\mathrm{a}}$ & $13.61 \pm 6.5^{\mathrm{a}}$ & $12.33 \pm 7.2^{\mathrm{a}}$ & $3.59 \pm 5.0^{\mathrm{a}}$ \\
\hline $\mathrm{BN}$ & 160 & $29.81 \pm 9.5^{b}$ & $23.34 \pm 5.7^{b}$ & $15.92 \pm 4.7^{b}$ & $17.16 \pm 7.1^{\mathrm{b}}$ & $12.40 \pm 5.2^{\mathrm{b}}$ \\
\hline BED & 217 & $40.10 \pm 12.1^{\mathrm{a}}$ & $36.43 \pm 6.9^{c}$ & $13.33 \pm 5.4^{\mathrm{a}}$ & $20.26 \pm 5.9^{c}$ & $11.04 \pm 5.0^{\mathrm{b}}$ \\
\hline EDNOS & 118 & $30.64 \pm 10.7^{\mathrm{b}}$ & $23.22 \pm 7.4^{\mathrm{b}}$ & $14.72 \pm 6.2^{\mathrm{ab}}$ & $14.96 \pm 7.2^{\mathrm{a}}$ & $5.62 \pm 5.3^{\mathrm{a}}$ \\
\hline Total & 562 & $33.53 \pm 12.0$ & $27.62 \pm 9.7$ & $14.40 \pm 5.6$ & $17.32 \pm 7.2$ & $9.41 \pm 6.1$ \\
\hline$F(3,554)$ & & $46.7^{* *}$ & $236.34^{* *}$ & $7.28^{* *}$ & $30.90^{* *}$ & $75.13^{* *}$ \\
\hline
\end{tabular}



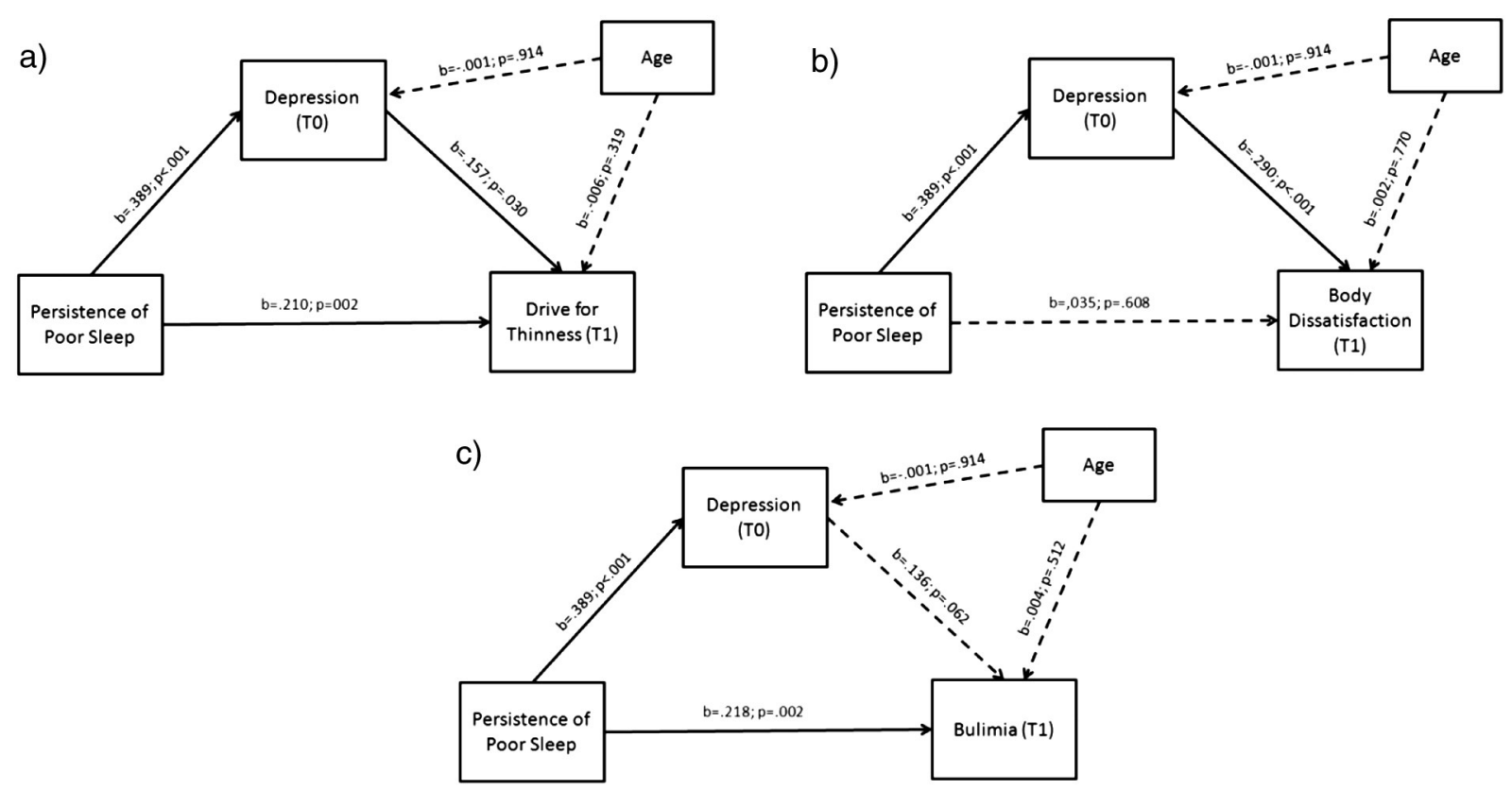

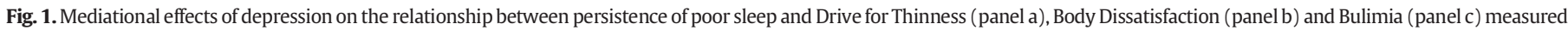
at $\mathrm{T} 1$ and controlling for age.

2) Persistent Poor Sleep group (PPSG): patients scoring above the median of the sample at both $\mathrm{T} 0$ and $\mathrm{T} 1$ assessments: $\mathrm{N}=88$;

3) Transient Poor Sleep group (TPSG): patients scoring above the median of the sample either at $\mathrm{T} 0$ or at $\mathrm{T} 1$ assessment: $\mathrm{N}=85$.

Sleep groups were thus compared for the GSI measured at T1 through a one-way ANOVA evidencing a significant difference across groups $\left(\mathrm{F}_{(2,237)}=23.41 ; \mathrm{p}<.001\right)$. Scheffé post-hoc tests indicated that the GSG group had lower GSI scores $(\mathrm{M}=.78$; $\mathrm{SD}=.48)$ than the other two groups (respectively PPSG: $\mathrm{M}=1.48$; $\mathrm{SD}=.70$; TPSG: $\mathrm{M}=1.25 ; \mathrm{SD}=.68$ ) that did not differ from each-other.

\section{Discussion}

The present study aimed to provide direct evidence of the existence of a relationship between sleep problems and severity of eating disorder symptoms and to assess whether this relationship is direct or is mediated by the relationship that both disorders share with depression. Both the direct and indirect relationships were evaluated cross-sectionally and longitudinally, after 6 months of standard treatment.

The findings are consistent with previous evidence indicating that sleep and eating disorders share a common variance (Kim et al., 2010; Lombardo et al., 2013; Soares et al., 2011), and that both share a common variance with depression (Daga et al., 2011; Staner, 2010). Furthermore, they indicate that the direct relationship is present only when poor sleep is chronically present. Namely, poor sleep measured at the first clinical assessment is not directly predictive of any measure of severity of the eating disorder symptoms either cross-sectionally or longitudinally although it indirectly predicts the severity of the clinical condition through the mediation of depression. However, when an index of persistence of poor sleep is used both the indirect and the direct links are significant.

Although the mediational models used in the present study imply causal relationships, supported by the longitudinal design, the results do not imply that sleep problems and depression are causes of the eating disorders. Instead they confirm that when poor sleep is present and persistent in patients with eating disorders, this persistence may promote either comorbidity with other psychiatric disorders (in the present case depression or other clinical condition determining an enhanced GSI score) or resistance to the standard treatment.

These results thus encourage taking care of sleep in the treatment protocols of eating disorders aiming at reducing sleep difficulties and related cognitive and emotional correlates of sleep loss in order to potentiate treatment efficacy of the main clinical disorder, as evidenced for other psychiatric conditions (Haynes, Parthasarathy, Kersh, \& Bootzin, 2011).

Before concluding, limitations of the present study should be acknowledged. First of all the study is an archive research and examined data collected for clinical and not for research purposes. This kind of research method is vulnerable to several methodological problems (e.g. Keppel, Saufley, \& Tokunaga, 1992) that weaken the strength of the results and reduce the possibility to generalize them. An example that may have affected the present study is the selective storage bias: for instance we measured poor sleep through the sleep subscales of the SCL-90 and we didn't have the possibility to assess reliability of that scale as we only had access to the scale scores not to the item scores. Moreover, both the severity of the eating disorders and poor sleep were assessed through psychometric instruments widely used in the clinical field but whose construct validity has been sometimes questioned (e.g. Arrindell, Barelds, Janssen, Buwalda, \& van der Ende, 2006; Maïano, Morin, Monthuy-Blanc, Garbarino, \& Stephan, 2009). Future studies should be planned and conducted using a more sensitive study design and more appropriate measures of sleep problems.

Notwithstanding these limitations the present study has also several strengths that merit to be addressed. The first is the wide sample of patients (more than 500); and the second is the longitudinal design.

\section{Role of funding sources}

The authors received no financial support for conducting the research and preparing the manuscript.

\section{Contributors}

Lombardo C. designed the study and wrote the protocol. Venezia C. and Salvemini V. extracted the data from the clinical files. Lombardo $\mathrm{C}$. and Battagliese G. conducted the statistical analyses and wrote the first draft of the manuscript. Salvemini V. was responsible for the protection of the patients' privacy. All authors contributed to the manuscript preparation and approved the final version. Lombardo C. provided the revised manuscript. 
Conflict of interest

None of the authors have conflicts of interest to disclose.

\section{References}

Arrindell, W.A., Barelds, D.P., Janssen, I.C., Buwalda, F.M., \& van der Ende, J. (2006) Invariance of SCL-90-R dimensions of symptom distress in patients with peri partum pelvic pain (PPPP) syndrome. British Journal of Clinical Psychology, 45, 377-391. http://dx.doi.org/10.1348/014466505X68924.

Bayard, S., \& Dauvilliers, Y.A. (2013). Reward-based behaviors and emotional processing in human with narcolepsy-cataplexy. Frontiers in Behavioral Neuroscience, 7, 1-7. http://dx.doi.org/10.3389/fnbeh.2013.00050.

Crisp, A.H., Stonehill, E., \& Fenton, G.W. (1971). The relationship between sleep nutrition and mood: A study of patients with anorexia nervosa. Postgraduate Medical Journal, 47, 207-213. http://dx.doi.org/10.1136/pgmj.47.546.207.

Daga, G.A., Gramaglia, C., Bailer, U., Bergese, S., Marzola, E., \& Fassino, S. (2011). Major depression and avoidant personality traits in eating disorders. Psychotherapy and Psychosomatics, 80, 319-320. http://dx.doi.org/10.1159/000324761.

Derogatis, L.R., Lipman, R.S., \& Covi, L. (1973). SCL-90: An outpatient psychiatric rating scale - Preliminary report. Psychopharmacological Bulletin, 9, 13-28.

Derogatis, L.R., Rickels, K., \& Rock, A.F. (1976). The SCL-90 and the MMPI: A step in the validation of a new self-report scale. British Journal of Psychiatry, 1, 280-289. http:// dx.doi.org/10.1192/bjp.128.3.280.

Garner, D.M. (1991). Eating Disorder Inventory-2: Professional Manual. Odessa: Psychological Assessment Resources.

Haynes, P.L., Parthasarathy, S., Kersh, B., \& Bootzin, R.R. (2011). Examination of insomnia and insomnia treatment in psychiatric inpatients. International Journal of Mental Health Nursing, 20, 130-136. http://dx.doi.org/10.1111/j.1447-0349.2010.00711.x.

Horowitz, L.M., Rosenberg, S.E., Baer, B.A., Ureno, G., \& Villasenor, V.S. (1988). Inventory of interpersonal problems: Psychometric properties and clinical applications. Journal of Consulting and Clinical Psychology, 56, 885-892. http://dx.doi.org/10.1037/0022006X.56.6.885.

Keppel, G., Saufley, W.H., \& Tokunaga, H. (1992). Introduction to Design and Analysis: A Students Handbook (2nd ed.). New York (NY): W.H. Freeman \& Company.

Kim, K.R., Jung, Y.C., Shin, M.Y., Namkoong, K., Kim, J.K., \& Lee, J.H. (2010). Sleep disturbance in women with eating disorders: Prevalence and clinical characteristics. Psychiatry Research, 176, 88-90. http://dx.doi.org/10.1016/j.psychres.2009.03.021.

Lauer, C.J., \& Krieg, J.C. (2004). Sleep in eating disorders. Sleep Medicine Reviews, 8, $109-118$
Lombardo, C., Battagliese, G., Baglioni, C., David, M., Violani, C., \& Riemann, D. (2013). Severity of insomnia, disordered eating symptoms, and depression in female university students. Clinical Psychologist. http://dx.doi.org/10.1111/cp.12023 (pub. Online 2 Sept. 2013).

Maïano, C., Morin, A.J.S., Monthuy-Blanc, J., Garbarino, J.M., \& Stephan, Y. (2009). Eating disorders inventory: Assessment of its construct validity in a nonclinical French sample of adolescents. Journal of Psychopathological Behavior, 31, 387-404. http:// dx.doi.org/10.1007/s10862-009-9128-2.

Makino, M., Hashizume, M., Yasushi, M., Tsuboy, K., \& Dennerstein, L. (2006). Factors associated with abnormal eating attitudes among female college students in Japan. Archives of Women's Mental Health, 9, 203-208. http://dx.doi.org/10.1007/s00737006-0128-x.

Ohno, K., \& Sakurai, T. (2008). Orexin neuronal circuitry: Role in the regulation of sleep and wakefulness. Frontiers in Neuroendocrinology, 29, 70-87. http://dx.doi.org/10. 1016/j.yfrne.2007.08.001.

Patel, S.R. (2009). Reduced sleep as an obesity risk factor. Obesity Review, 10, 61-68. http://dx.doi.org/10.1111/j.1467-789X.2009.00664.x.

Patel, S.R., Blackwell, T., Redline, S., Ancoli-Israel, S., Cauley, J.A., \& Hillier, T.A. (2008). The association between sleep duration and obesity in older adults. International Journal of Obesity, 32, 1825-1834. http://dx.doi.org/10.1038/ijo.2014.13.

Preacher, K.J., \& Hayes, A.F. (2008). Asymptotic and resampling strategies for assessing and comparing indirect effects in multiple mediator models. Behavior Research Methodology, 40, 879-891. http://dx.doi.org/10.3758/BRM.40.3.879.

Soares, M. J., Macedo, A., Bos, S. C., Maia, B., Marques, M., Pereira, A. T., Gomes, A. A., Valente, J., Nogueira, V., \& Azevedo, M. H. (2011). Sleep disturbances, body mass index and eating behaviour in undergraduate students. Journal of Sleep Research, 20, 479-486. http://dx.doi.org/10.1111/j.1365-2869.2010.00887.x.

Staner, L. (2010). Comorbidity of insomnia and depression. Sleep Medicine Reviews, 14, 35-46. http://dx.doi.org/10.1016/j.smrv.2009.09.003.

Van Cauter, E., \& Knutson, K.L. (2008). Sleep and the epidemic of obesity in children and adults. European Journal of Endocrinology, 159, S59-S66. http://dx.doi.org/10.1530/ EJE-08-0298.

Van der Berg, J.F., Knvistingh Neven, A., Tulen, J.H., Hofman, A., Witteman, J.C., Miedema, H.M., et al. (2008). Actigraphic sleep duration and fragmentation are related to obesity in the elderly: The Rotterdam study. International Journal of Obesity, 32, 1083-1090. http://dx.doi.org/10.1038/ijo.2008.57. 